\title{
Impact of performance interdependencies on structural vulnerability: a systems perspective of storm surge risk to coastal residential communities
}

\author{
Adam Hatzikyriakou ${ }^{\mathrm{a}}$, Ning Lin ${ }^{\mathrm{b}}$
}

\begin{abstract}
\footnotetext{
${ }^{a}$ Corresponding Author

Dept. of Civil and Environmental Engineering

Princeton University

Engineering Quad E218

Princeton, NJ 08540, United States

201-566-8058

ah7@princeton.edu

${ }^{\mathrm{b}}$ Dept. of Civil and Environmental Engineering

Princeton University

Engineering Quad E328

Princeton, NJ 08540, United States

609-258-0266

nlin@princeton.edu
}

Interaction between residential structures during natural hazards can lead to interdependencies in their performance. During storm surge, for example, structures can affect the performance of inland buildings by generating damaging waterborne debris or by beneficially dampening surge loads. Quantifying the impact of this interaction on structural vulnerability is critical for risk assessment and informed decision-making. In this study we present and implement two general modeling approaches for investigating such interdependencies. The first method is to condition the vulnerability of a structure on the performance of neighboring buildings using a Markov model. The second uses a marginal model to account for correlation between damage observations when estimating a structure's vulnerability to the hazard. Both approaches are implemented using a case study of an impacted coastal community during Hurricane Sandy (2012). Findings indicate that a structure's performance during storm surge is strongly dependent on the damage state of the structure immediately seaward. Furthermore, considering the correlated damage states of buildings increases statistical uncertainty when relating structural performance to hazard intensity. Motivated by these findings, we propose a more coordinated approach to coastal risk mitigation which considers the effects of interdependencies on insurance pricing, structural design, mitigation strategies and community resilience. 


\section{Keywords}

performance interdependencies, system reliability, storm surge, Hurricane Sandy, residential structures, community resilience

\section{Introduction}

Assessing the risk which natural hazards pose frequently relies on estimating the vulnerability of structures to physical damage or economic losses. This estimation is commonly done by statistically relating the assessed performance of structures following hazard events to their estimated hazard exposure. Common assessments of structural performance include binary classifications of failure and survival for investigating limit-state performance or more descriptive assessments based on multiple ordinal damage states (e.g. 0-25\%, 25-50\%, 50-75\%, $75-100 \%$ damage). The analysis yields commonly used vulnerability and fragility curves, which respectively give the probability of a structure being damaged or exceeding a damage state as a function of hazard intensity.

Vulnerability estimation, however, can be complicated when a structure's exposure to a hazard is compounded by interdependencies with the performance of other structures. This is oftentimes the case with infrastructure systems such as energy lifelines and communication networks, where physical interaction between system components acts as an additional demand parameter affecting their performance [1]. As a result, failure of one component can magnify the vulnerability of connected components and in turn significantly affect the overall vulnerability of the system. The overload of one of the highly coupled stations and transmission lines comprising electric power grids, for example, can result in cascading outages across the entire grid, as illustrated by the 2003 North American Blackout [2]. Although infrastructures systems like power grids can be extremely complex, performance interdependencies can often be physically modeled since the interaction mechanisms are either clearly defined or can be reasonably assumed. Among a variety of modeling methods, two popular approaches include treating components as agents interacting according to prescribed rules or modeling their topology as a network of interconnected nodes [3]. The latter approach has been used to develop so-called 'interdependent fragility curves,' relating the loss of connectivity between network nodes to the magnitude of their interdependence and hazard intensity [4].

Performance interdependencies, however, are not unique to components of large infrastructure systems. For many hazards, similar effects can likewise be observed on the scale of neighboring buildings within residential communities. During earthquakes, for example, this interaction can 
occur due to the physical pounding of adjacent structures damaged by seismic excitation [5]. Interdependencies can likewise arise during windstorms when pressure damage to a structure generates windborne debris which impact and damage neighboring buildings [6]. A similar dynamic occurs during fires following earthquakes where ignitions caused by seismic damage induce the spread of fire between structures [7]. Performance interdependencies are also common features of many hydrological hazards including riverine flooding, tsunamis, and the focus of this study, storm surge [8-10]. Two interaction mechanisms contributing to these interdependencies are structural shielding and waterborne debris. The former occurs when the presence of a structure dampens surge loads on surrounding buildings and the latter when poorly performing buildings generate debris that damage inland structures.

The effects of storm surge from Hurricane Sandy (2012) in heavily impacted Ortley Beach, New Jersey, shown before and after Sandy in Figure 1, illustrate the modeling challenges presented by these performance interdependencies. Located on a low lying barrier island with a susceptible coastal dune, Ortley Beach experienced significant inundation during Sandy [11]. In particular, a hindcast simulation of hydrodynamic conditions in the community found that among hazard characteristics, maximum wave force potential (a surge parameter combining inundation with flow velocity) was a strong predictor of surveyed damage [12]. This hazard exposure, however, was likewise compounded by substantial interaction between structures. Tomizek et al. [12], for example, found that the number of surviving structures seaward of a building was strongly related to its damage state. Furthermore, Hatzikyriakou et al. [10] noted that waterborne debris significantly contributed to structural damage throughout the community. These observations indicate that far from being a simplistic collection of buildings, residential coastal communities comprise infrastructure systems whose overall reliability depends on the complex interaction of individual structures.

Motivated by previous studies of infrastructure systems, one potential approach to dealing with performance interdependencies within residential communities is by directly modeling the physical interaction between structures. Shielding effects, for example, could be considered by accounting for the presence of structures when simulating the spatially correlated loading on buildings. Furthermore, simplifying assumptions regarding debris generation and trajectory could be used to physically model debris interaction $[13,14]$. In practice, however, accounting for the presence of buildings when numerically simulating loads is computationally expensive if possible and physically modeling debris interaction is prone to significant uncertainties. The inevitable lack of information about the interaction between structures often means that the 
effects of performance interdependencies need to be statistically inferred from observed damage to structures. Although studies such as those of Hurricane Sandy and others have acknowledged the effects of performance interdependencies on residential structures, means of statistically quantifying its impact on structural vulnerability and the implications on risk assessment remain to be explored.

We address both of these objectives in this study by presenting and implementing statistical models for investigating the two critical aspects of performance interdependencies, namely (1) how a structure's performance depends on the performance of neighboring buildings and (2) how accounting for this dependence affects the interpretation of the structure's fragility to the hazard. To answer the first question we explicitly model a structure's vulnerability to performance interdependencies in addition to its vulnerability to the hazard. This is done with a Markov model by conditioning the performance of a structure on hazard covariates as well as on the performance of neighboring buildings. This analysis yields a set of vulnerability and fragility curves relating a structure's performance to hazard intensity conditional on the performance of surrounding buildings. To address the second question we use a marginal model to account for the correlated damage states of nearby buildings when statistically relating structural performance to the hazard. This analysis yields vulnerability and fragility curves which implicitly incorporate the effects of the interdependencies.

We begin in Section 2 by introducing vulnerability estimation when assuming independence using conventional logistic regression. This initial framework is then extended to consider interdependencies using the two proposed models. The implementation of each is illustrated in Section 3 using a case study of storm surge damage in Ortley Beach due to Hurricane Sandy. Motivated by previous studies, we consider the effects of wave force potential and performance interdependencies on assessed physical damage and economic losses. In Section 4 we discuss the implication of our findings on storm surge risk assessment by proposing a systems approach to coastal community resilience. Finally, in Section 5 we summarize and discuss potential extensions of the presented modeling approaches and propose directions for future research.

\section{Methodology Development}

To motivate the vulnerability estimation methods to follow, consider a hypothetical coastal community shown in Figure 2. Given the regular layout typical of many communities (e.g. Ortley Beach; Figure 1), the location of each structure relative to surrounding buildings can be uniquely defined by first grouping structures into $I$ clusters according to an index $i$. An 
additional index $j$ denotes the row of each structure from the coast, with $n_{i}$ representing the number of structures in cluster $i$ (i.e. the clusters may have different length). Following an event, each structure's performance level, whether in terms of physical damage or economic losses, can be classified using a binary failure/survival assessment or using multiple percentage-based ordinal damage states. A set of hazard covariates describing each structure's exposure can likewise be estimated using data (e.g. gauge measurements and high water marks) or hindcast simulations of hydrodynamic conditions.

Ignoring the potentially correlated performance of nearby structures, existing vulnerability studies have traditionally assumed the independence of damage observations when empirically relating performance to hazard covariates. A standard approach for modeling independent binary (and by extension ordinal) data is using logistic regression. Examples of studies using logistic regression to estimate structural vulnerability include Hatzikyriakou et al. [10] for storm surge and Reese et al. [15] for tsunami. Taking advantage of the presumed independence of observations (and thus ignoring the indexing in Figure 2 as all observations are considered independent and identically distributed), a convenient maximum likelihood function can be derived and numerically solved to estimate regression parameters. In cases where observations are in fact correlated, however, the typical consequence of assuming independence is deflated estimates of parameter standard errors or incorrect parameter estimates altogether [16, 17]. More precisely, when correlation between observations is positive (negative), ignoring correlation results in an underestimation (overestimation) of parameter variances [18].

One approach to dealing with performance interdependencies is by treating interaction between structures as an additional demand parameter. This can be done with a Markov model by explicitly conditioning the performance of structures on that of neighboring buildings along with hazard covariates. The indexing of structures noted in Figure 2 can be used to relate nearby buildings, with the particular choice of conditioning motivated by physical intuition or previous experience. In the case of storm surge, where flow velocity and wave impact are greatest in the on-shore direction, conditioning the performance of a structure $(i, j)$ on the performance of the structure immediately seaward $(i, j-1)$ appears reasonable. Given this conditioning, the performance of structures can be treated as conditionally independent and the same maximum likelihood approach as for logistic regression can be used to fit the model [19]. Similar spatial Markov-based approaches have seen extensive use in ecological studies of forest vulnerability where the spread of diseases between nearby trees results in their correlated survival [20, 21]. Markov models have likewise been integral in the study of structural performance over time, 
where conditioning is based on a structure's previous performance history. Examples include the deterioration of wastewater systems and structures exposed to multiple earthquakes [22, 23].

Instead of modeling a structure's conditional vulnerability, one can also develop an unconditional vulnerability function that appears in traditional form but implicitly accounts performance interdependencies. This can be done using a marginal model, so called because the effects of hazard covariates on the marginal (i.e. mean) damage response are modeled separately from the correlation between observations. Once again using the $(i, j)$ indexing as a means of relating neighboring buildings, interdependencies are dealt with by modeling the correlated performance of structures within each cluster $i$. Using the same motivation as for the conditioning used in the Markov model, one may assume that only structures in adjacent rows are correlated. This marginal model approach extends the logistic regression framework and leads to modified equations for estimating regression parameters and their standard errors [24]. Marginal models have been used to analyze spatial correlation of the frequency of hazards, such as extreme rainfall events [25]. Furthermore, marginal models have seen widespread use in the analysis of correlated longitudinal data, which consist of repeated observations on individual subjects collected over time [26].

Following this general introduction of each modeling approach, we next present the technical details of each for both binary and ordinal data. We note functions for implementing each method in R, a commonly used statistical software [27]. Each of the methods can similarly be implemented in MATLAB.

\subsection{Logistic Regression}

Ignoring the $(i, j)$ indexing relating neighboring structures when considering interdependencies, let $Y_{k}$ and $\boldsymbol{x}_{\boldsymbol{k}}=\left(x_{k, 1}, x_{k, 2}, \ldots, x_{k, p}\right)^{\prime}$ respectively represent a dichotomous damage response (coded 1 for failure, 0 for survival) and a vector of $p$ observed explanatory variables for structure $k=1,2, \ldots, n$, where $n$ is the total number of structures in the community. The mean of response $Y_{k}$, which is equivalent to the probability of observing failure, may be related to $\boldsymbol{x}_{\boldsymbol{k}}$ in a logistic regression

$$
\mu_{k}=E\left[Y_{k} \mid \boldsymbol{x}_{k}\right]=P\left[Y_{k}=1 \mid \boldsymbol{x}_{k}\right]=\frac{e^{\alpha+x_{k}^{\prime} \boldsymbol{\beta}}}{1+e^{\alpha+x_{k}^{\prime} \boldsymbol{\beta}}}
$$

which can be transformed into a linear predictor using a link function logit $(\cdot)$, 


$$
\operatorname{logit}\left(\mu_{k}\right)=\log \frac{\mu_{k}}{1-\mu_{k}}=\alpha+\boldsymbol{x}_{\boldsymbol{k}}^{\prime} \boldsymbol{\beta}
$$

where $\alpha$ is a regression intercept and $\boldsymbol{\beta}=\left(\beta_{1}, \ldots, \beta_{p}\right)^{\prime}$ is a vector of regression coefficients. Given a sample of observations, $\boldsymbol{y}=\left(y_{1}, \ldots, y_{n}\right)^{\prime}$, the regression parameters $\boldsymbol{\theta}=\left(\alpha, \boldsymbol{\beta}^{\prime}\right)^{\prime}=$ $\left(\theta_{0}, \theta_{1}, \ldots, \theta_{p}\right)$ can be estimated using maximum likelihood. Given the assumed independence of damage observations, a likelihood function can be defined as

$$
\mathcal{L}(\boldsymbol{\theta})=\prod_{k=1}^{n} f\left(y_{k}\right)
$$

where $f\left(y_{k}\right)$ represents the probability of observing the $k^{t h}$ observation and

$$
f\left(y_{k}\right)=\left(\mu_{k}\right)^{y_{k}}\left(1-\mu_{k}\right)^{1-y_{k}}
$$

Using an iteratively reweighted least squares algorithm (the function $\mathrm{g} l \mathrm{~m}$ in $\mathrm{R}$ ), maximum likelihood estimates for regression parameters, $\widehat{\boldsymbol{\theta}}$, and their covariance matrix, $\widehat{\operatorname{Var}}[\widehat{\boldsymbol{\theta}}]$, can be obtained. The variance $\left(\widehat{\operatorname{Var}}\left[\hat{\theta}_{s}\right]\right)$ and covariance $\left(\widehat{\operatorname{Cov}}\left[\hat{\theta}_{s}, \hat{\theta}_{t}\right]\right)$ of regression parameters are respectively the diagonal and off-diagonal terms of the covariance matrix. Then, for a vector $\boldsymbol{x}=\left(x_{0}, x_{1}, x_{2}, \ldots, x_{p}\right)^{\prime}$ representing a set of values for the $p$-covariates (where $x_{0}=1$ corresponding to the regression intercept), the estimator of $\operatorname{logit}(\mu(\boldsymbol{x}))$, denoted as $\widehat{\operatorname{logit}}(\boldsymbol{x})$, is

$$
\widehat{\operatorname{logit}}(\boldsymbol{x})=\boldsymbol{x}^{\prime} \widehat{\boldsymbol{\theta}}
$$

which can be transformed back to obtain the estimator of the failure probability (Eq. 1). An estimator of the standard error of $\widehat{\operatorname{logit}}(\boldsymbol{x})$ can be computed as

$$
\widehat{S E}[\widehat{\operatorname{logit}}(\boldsymbol{x})]=\sqrt{\sum_{s=0}^{p} x_{s}^{2} \widehat{\operatorname{Var}}\left[\hat{\theta}_{s}\right]+\sum_{s=0}^{p} \sum_{t=s+1}^{p} 2 x_{s} x_{t} \widehat{\operatorname{Cov}}\left[\hat{\theta}_{s}, \hat{\theta}_{t}\right]}
$$

The $100(1-\alpha) \%$ Wald-based confidence intervals for the failure probability based on the asymptotic normality of parameter estimators is then given by

$$
\left[p_{L}, p_{U}\right]=\frac{\exp \left\{\widehat{\operatorname{logit}}(\boldsymbol{x}) \pm z_{1-\alpha / 2} \widehat{S E}[\widehat{\operatorname{logit}}(\boldsymbol{x})]\right\}}{1+\exp \left\{\widehat{\operatorname{logit}}(\boldsymbol{x}) \pm z_{1-\alpha / 2} \widehat{S E}[\widehat{\operatorname{logit}}(\boldsymbol{x})]\right\}}
$$

where $p_{L}$ and $p_{U}$ are lower and upper confidence bounds and $z_{1-\alpha / 2}$ is the $1-\alpha / 2$ percentile of the standard normal distribution. 
In the case of ordinal damage assessments, the performance of a structure $Y_{k}$ can take one of $D$ discrete states labeled $0,1, \ldots, d, \ldots, D-1$. To preserve the ordered nature of the damage scale, the cumulative probability of a damage state $P\left[Y_{k} \leq d\right]$ is typically the quantity of interest. One modeling approach is to dichotomize the ordinal data into binary responses ( 1 if $y_{k} \leq d$ and 0 if $y_{k}>d$ ) and to fit separate logistic regression models for $d=0,1, \ldots, D-2$ (note $P\left[Y_{k} \leq D-\right.$ $1=1$ ). This dichotomization, however, does not take complete advantage of the ordinal nature of the damage states and can lead to an excessive amount of regression parameters to be interpreted. A popular alternative is the cumulative logit model, which is given by

$$
\operatorname{logit}\left(P\left[Y_{k} \leq d\right]\right)=\alpha_{d}+\boldsymbol{x}_{\boldsymbol{k}}^{\prime} \boldsymbol{\beta} \quad d=0,1, \ldots, D-2
$$

In this model the effects of the covariates are assumed to be the same for all damage states whereas the intercept $\left\{\alpha_{d}\right\}$ is allowed to differ [28, 29]. As with standard logistic regression, parameters in the cumulative logit model $\boldsymbol{\theta}=\left(\left\{\alpha_{d}\right\}, \boldsymbol{\beta}^{\prime}\right)^{\prime}$ can be estimated with maximum likelihood (using the function vglm or polr in R).

\subsection{Markov Model}

Reintroducing the $(i, j)$ indexing from Figure 2, the Markov model conditions the performance of a structure on the performance of neighboring buildings in addition to hazard covariates. In the general case, we assume that the response of a structure $Y_{i, j}$ is conditionally dependent on $\mathcal{H}_{i, j}$, or the response of the $q$ structures immediately seaward.

$$
\mathcal{H}_{i, j}=\left\{y_{i, j-1}, \ldots, y_{i, j-q}\right\}
$$

The conditional mean response of a structure in cluster $i$ and row $j, \mu_{i, j}^{C}=E\left[Y_{i, j} \mid \mathcal{H}_{i, j}, \boldsymbol{x}_{i, j}\right]$, is taken to satisfy the equation

$$
\operatorname{logit}\left(\mu_{i, j}^{C}\right)=\alpha+\boldsymbol{x}_{i, j}^{\prime} \boldsymbol{\beta}+h\left(\mathcal{H}_{i, j} ; \boldsymbol{\gamma}\right)
$$

where $h(\cdot)$ is a function of the neighboring responses $\mathcal{H}_{i, j}$ and an additional vector of regression coefficients $\boldsymbol{\gamma}$. As noted earlier, a simple yet physically intuitive Markov model for storm surge is to linearly condition only on the response of the structure immediately seaward (i.e. $\mathcal{H}_{i, j}=$ $\left\{y_{i, j-1}\right\}$ ) according to

$$
\operatorname{logit}\left(\mu_{i, j}^{C}\right)=\alpha+\boldsymbol{x}_{i, j}^{\prime} \boldsymbol{\beta}+y_{i, j-1} \gamma
$$

In this Markov model, regression coefficients $\boldsymbol{\beta}$ can be interpreted as the effect of each hazard covariate holding other covariates and the response of the seaward structure constant. 
Furthermore, hazard covariates are taken to have the same effect on the response $Y_{i, j}$ regardless of the neighboring response $y_{i, j-1}$. The differing effects of covariates could be considered by including additional interaction terms $\gamma_{P} x_{i, j, P} y_{i, j-1}$ for each of the $P=1, \ldots, p$ explanatory variables. Depending on the number of covariates considered, however, interaction effects can introduce a large number of additional parameters which may be problematic when constrained by limited data.

Regression parameters $\boldsymbol{\theta}=\left(\alpha, \boldsymbol{\beta}^{\prime}, \gamma\right)^{\prime}$ can be estimated by first noting that the joint distribution of a set of observations in a cluster $\left(y_{i, 1}, \ldots, y_{i, n_{i}}\right)$ can be decomposed into a product of terms

$$
f\left(y_{i, 1}, \ldots, y_{i, n_{i}}\right)=f\left(y_{i, 1}\right) \prod_{j=2}^{n_{i}} f\left(y_{i, j} \mid y_{i, j-1}\right)
$$

given that each response is conditioned only on the response immediately seaward.

Although the distribution $f\left(y_{i, j} \mid y_{i, j-1}\right)$ is specified by the choice of $\operatorname{logit}\left(\mu_{i, j}^{C}\right)$ (as $f\left(y_{i, j} \mid y_{i, j-1}\right)=\left(\mu_{i, j}^{C}\right)^{y_{i, j}}\left(1-\mu_{i, j}^{C}\right)^{1-y_{i, j}}$ ), the distribution of $f\left(y_{i, 1}\right)$ is left unspecified by the model and therefore a full likelihood function cannot be defined. In this case, we choose the estimate of $\boldsymbol{\theta}$ which maximizes the conditional likelihood

$$
\mathcal{L}^{\mathcal{C}}(\boldsymbol{\theta})=\prod_{i=1}^{I} f\left(y_{i, 2}, \ldots, y_{i, n_{i}} \mid y_{i, 1}\right)=\prod_{i=1}^{I} \prod_{j=2}^{n_{i}} f\left(y_{i, j} \mid y_{i, j-1}\right)
$$

Although this conditional likelihood requires that damage observations from the first row of each cluster be removed from the analysis, the linearity of $\operatorname{logit}\left(\mu_{i j}^{C}\right)$ with respect to the regression parameters means that the Markov model can be fit in the same fashion as standard logistic regression, with the response $Y_{i, j}$ regressed against the vector of explanatory variables $\left(\boldsymbol{x}_{\boldsymbol{i}, j}, y_{i, j-1}\right)$.

For ordinal damage states, the cumulative logit model can be extended to condition the performance of a structure on the multiple discrete damage states of neighboring structures. A first-order Markov model proposed by Diggle et al. (2002) can be written as

$$
\operatorname{logit}\left(P\left[Y_{i, j} \leq d \mid Y_{i, j-1}=y_{i, j-1}\right]\right)=\alpha_{d}+\boldsymbol{x}_{i, j}^{\prime} \boldsymbol{\beta}+\widetilde{\boldsymbol{y}}_{i, j-1}^{\prime} \boldsymbol{\gamma} \quad d=0,1, \ldots, D-2
$$

Performance dependence on the seaward building is introduced with $\tilde{\boldsymbol{y}}_{i, j-\mathbf{1}}=\left(\tilde{y}_{i, j-1,0}, \tilde{y}_{i, j-1,1}, \ldots, \tilde{y}_{i, j-1, D-2}\right)^{\prime}$ and the regression coefficients $\boldsymbol{\gamma}=\left(\gamma_{0}, \ldots, \gamma_{D-2}\right)^{\prime}$. The derived variable $\widetilde{\boldsymbol{y}}_{i, j-1}$ is comprised of indicator variables defined according to 


$$
\tilde{y}_{i, j, d^{\prime}}=\left\{\begin{array}{lr}
1 & \text { if } y_{i, j-1} \leq d^{\prime} \\
0 & \text { otherwise }
\end{array}\right.
$$

which define the damage state of the seaward building. In the case of four damage states $(D=4)$ for example, Table 1 illustrates how $y_{i, j-1}$ and $\tilde{\boldsymbol{y}}_{\boldsymbol{i}, \boldsymbol{j}-\mathbf{1}}$ are related. Following the same logic as the binary Markov model, the specific choice of conditioning allows for a likelihood function consisting of a product of conditionally independent terms to be defined [30].

\subsection{Marginal Model}

Rather than explicitly conditioning on the performance of neighboring buildings, the marginal model approach implicitly considers interdependencies by incorporating the statistical correlation of damage observations into the estimation of regression parameters. As with logistic regression, the mean damage response $\mu_{i, j}$ is related to hazard covariates according to

$$
\operatorname{logit}\left(\mu_{i, j}\right)=\alpha+\boldsymbol{x}_{i, j}^{\prime} \boldsymbol{\beta}
$$

The effects of interdependencies are treated by considering the correlated response of structures within each cluster, which is modeled using a correlation matrix $\boldsymbol{R}_{i}=\left[\rho_{j, k}\right]$ whose elements $\rho_{j, k}=\operatorname{cor}\left(Y_{i, j}, Y_{i, k}\right)$ are the pairwise correlations between structural responses in cluster $i$. Although the correlation matrix can potentially differ from cluster to cluster, it is typically treated as constant, representing the average dependence amongst structures over all clusters [31]. The choice of $\boldsymbol{R}_{i}$ is motivated by the context of problem and desire to make the model as parsimonious as possible. As with the Markov model described earlier, it is assumed that in the case of storm surge only adjacent structures in a cluster are correlated. An additional simplifying assumption is that this correlation is constant regardless of row, or more formally,

$$
\operatorname{cor}\left(Y_{i, j}, Y_{i, k}\right)=\rho \quad\{\forall j, k:|j-k|=1\}
$$

where $\rho$ is a regression parameter which can be estimated in addition to $\left(\alpha, \boldsymbol{\beta}^{\prime}\right)^{\prime}$. An alternative approach to modeling $\boldsymbol{R}_{i}$ is to allow the correlation of adjacent structures to differ with row. This choice of $\boldsymbol{R}_{i}$, however, can add a large number of parameters to the model and can be numerically difficult to estimate [24].

Unlike standard logistic regression where the assumption of independence allows for a convenient likelihood function comprised of a product of each observation's likelihood contribution, accounting for correlation can make defining a likelihood function complicated or intractable [18]. In the absence of a likelihood function, a quasi-likelihood approach can be taken which requires specification of only the mean and variance of $Y_{i, j}$ rather than its full distribution, 
resulting in so-called generalized estimating equations [24]. Regression parameters $\boldsymbol{\theta}=$ $\left(\alpha, \boldsymbol{\beta}^{\prime}, \rho\right)^{\prime}$ can be estimated by iterating between solving the generalized estimating equations for $\left(\alpha, \boldsymbol{\beta}^{\prime}\right)^{\prime}$ and estimating $\rho$ using residuals [32]. Estimates of $\left(\alpha, \boldsymbol{\beta}^{\prime}\right)^{\prime}$ are consistent (i.e. they converge in probability to the true parameters) even if the form of the correlation matrix is incorrectly specified [33]. The marginal model can be implemented in $\mathrm{R}$ using the function gee.

The standard errors of $\left(\alpha, \boldsymbol{\beta}^{\prime}\right)^{\prime}$ can be estimated using two approaches. Model-based standard errors assume that the chosen $\boldsymbol{R}_{i}$ is in fact the true correlation structure of observations (although the correlation parameters themselves are estimated from the data). Empirical standard errors are modifications of model-based estimates which make use of the observed correlation structure of observations. Although empirical standard errors are consistent even if $\boldsymbol{R}_{i}$ is not correctly specified, they can be very poor for small samples [34]. When the number of clusters is small (less than 20), therefore, it is recommended to used model-based estimators [35]. As with standard logistic regression, the distribution of regression parameter estimates obtained by solving the generalized estimation equations are asymptotically normal. Confidence intervals for the failure probability, therefore, can be obtained using Eq. 7 with either model or empiricallybased estimates of regression parameter standard errors.

A similar modeling approach can be taken for ordinal data by integrating the correlation of damage responses into the estimation of the cumulative logit model

$$
\operatorname{logit}\left(P\left[Y_{i, j} \leq d\right]\right)=\alpha_{d}+\boldsymbol{x}_{\boldsymbol{i}, \boldsymbol{\beta}}^{\prime} \boldsymbol{\beta} \quad d=0,1, \ldots, D-2
$$

Doing so requires specification of $\operatorname{cor}\left(Y_{i, j, d}, Y_{i, k, d^{\prime}}\right)$, which represents the correlation of each pair of damage levels $\left(d, d^{\prime}\right)$ for each pair of structures $(j, k)$ in a cluster $i$. The large number of additional regression parameters, however, may make numerically fitting the model difficult when there are not sufficient observations of each damage level. More details on fitting the model using generalized estimating equations can be found in Agresti et al. [17].

\section{Case Study}

We illustrate the implementation of the logistic, Markov and marginal models using a case study of Ortley Beach, New Jersey, a coastal community heavily impacted by storm surge from Hurricane Sandy. Our analysis is based on an assessment of physical damage and economic losses to structures in the community and a hindcast simulation of overland hydrodynamic conditions due to Sandy's surge.

\subsection{Hurricane Sandy}


Hurricane Sandy (2012) was a late season tropical cyclone which significantly impacted areas across the Atlantic coast with a devastating storm surge. Coastal communities in New Jersey and New York were particularly hard hit, with a record high storm tide elevation of 3.47 meters above NAVD88 observed at the Battery in New York City [36]. The resulting impact of surge on coastal infrastructure was devastating, with at least 650,000 houses either damaged or destroyed and total losses exceeding $\$ 50$ billion [36].

\subsection{Ortley Beach}

Located on a low-lying barrier island prone to inundation, the coastal community of Ortley Beach, New Jersey was one of the worst hit areas by Sandy's surge. Out of 2,772 structures in the primarily residential area, 705 (25\%) experienced losses greater than $\$ 10,000$ [37]. A U.S. Geological Survey (USGS) high-water mark gauge located 500 meters inland recorded an inundation (water level above ground) of $0.9 \mathrm{~m}$ [36]. Nearer to the coast, high water marks on damaged structures place inundation as high as 3 meters. A plan view image of the most impacted region of Ortley Beach before and after Hurricane Sandy is shown in Figure 1a and Figure $1 \mathrm{~b}$ respectively.

\subsection{Performance Interdependencies}

Following Hurricane Sandy, structures in Ortley Beach were surveyed by a group of students and faculty from the University of Notre Dame and Princeton University [10, 12, 38]. During the survey it was commonly observed that damaged structures were surrounded by buildings with similar damage states. A closer inspection reveals that waterborne debris and structural shielding were two mechanisms contributing to this correlated performance.

An extreme case of debris damage is illustrated in Figure 3a, which shows four damaged structures and their original footprints outlined and shaded with their respective color. The figure depicts an oceanfront structure outlined in red carried by the surge nearly a hundred meters until it impacted the structure outlined in blue, which in turn impacted the green structure and finally the orange structure. More generally, the size of debris and the resulting magnitude of damage varied widely, as shown in Figure 3b.

In contrast to these negative performance interdependencies, the presence of resilient structures was found to be beneficial to inland buildings by shielding them against debris and surge impact. Such constructive interdependencies have likewise been observed during other hydrological events. Following the 2002 Stromboli tsunami in the Aeolian archipelago, for example, shielding 
due to vegetation and other obstructions was noted as having reduced damage to structures [39]. Numerical simulations of inland inundation have also shown that coastal structures can dampen the effects of surge by reducing flow velocity [40].

\subsection{Damage Assessment}

The performance of structures in Ortley Beach was assessed according to both physical damage and economic losses. Using photographs of damage to structural components such as foundations, exterior walls and exterior siding, Hatzikyriakou et al. [10] assessed component performance on a binary failure/survival scale. Failure was defined as a component which no longer performed its intended structural purpose. With foundations being a critical component during storm surge and also exhibiting performance interdependencies (e.g. Figure 3a), we choose it as an indicator of the physical performance of structures. The results of the foundation assessment for Ortley Beach are shown in Figure 4a.

Using the same survey, Xian et al. [38] assessed physical damage to structural components on a percentage scale. To develop a metric of overall performance, a loss ratio (i.e. the percent loss in a structure's value) was derived for each surveyed building by weighing the percent damage to components by their estimated cost [38]. Assessed loss ratios for structures in Ortley Beach are shown in Figure 4b. For the purpose of this study, loss ratios have been converted into discrete damage states. To dichotomize loss ratios into a binary response, failure (survival) is defined according to whether a structure's loss ratio is greater (less) than $50 \%$. This criterion is chosen since FEMA requires that structures with 'substantial damage' (defined as greater than 50\% loss in market value) be brought to compliance with current standards following an event [41]. Loss ratios have likewise been converted into four ordinal damage states $(D=4)$ defined based on loss ratio intervals of $0-25 \%, 25-50 \%, 50-75 \%$ and $75-100 \%$. The damage states are coded 0,1 , 2 and 3 respectively.

\subsection{Hydrodynamic Simulation}

A hindcast of coastal inundation in Ortley Beach during the peak hour of Sandy's surge was simulated using a 2-Dimensional Boussinesq-Green-Naghdi model by Tomizcek et. al [12]. Peak surge, significant wave height and peak wave period used as model inputs were estimated from a surge-wave simulation of Hurricane Sandy using the coupled ADvanced CIRCulation (ADCIRC) and Simulating WAves Nearshore (SWAN) models. The model was implemented using post-Sandy bathymetry and topography to reproduce the worst possible hydrodynamic conditions during Sandy. Due to the numerical complexity of accounting for the effects of 
buildings on wave transformation, a bare-earth topography with buildings removed was used. The use of a "bare-earth" topography, which is typical of most wave modeling studies, proves beneficial for the purpose of this study since our goal is to investigate the respective contributions of storm surge and performance interdependencies on structural damage. The absence of buildings in the hydrodynamic simulation allows for an explicit separation of these two factors in the statistical modeling described earlier.

Storm surge exposure when estimating flood losses to structures has traditionally been characterized solely by inundation depth using depth-damage curves. However, additional surge features such as wave and hydrodynamic action also need to be considered. To incorporate these effects into a single storm surge metric, we instead characterize surge exposure using wave force potential, $F_{\text {wave }}(t)$, defined as

$$
F_{\text {wave }}(t)=d(t) u(t)^{2}
$$

where $d(t)$ and $u(t)$ are respectively time histories of overall surge inundation (including wave height) and on-shore velocity simulated at each building location. Wave force potential has previously been noted as a strong predictor of storm surge damage [12]. Time histories of inundation, on-shore velocity and wave force potential for a single structure are shown in Figure 5 . For each structure, the maximum simulated wave force potential $\left(F_{\text {wave,max }}\right)$ is extracted.

\subsection{Markov Model}

The dependence of a structure's vulnerability on maximum wave force potential and the performance of the structure immediately seaward is investigated using a first-order Markov model (Section 2.2). Two separate models using assessed foundation failure and loss failure as the damage response $Y_{i, j}$ are fit, with the estimated conditional probability of failure given by

$$
P\left[Y_{i, j}=1 \mid Y_{i, j-1}=y_{i, j-1}, F_{\text {wave } \text { max }}\right]=\frac{e^{\alpha+\beta \cdot F_{\text {wave }, \text { max }}+\gamma \cdot y_{i, j-1}}}{1+e^{\alpha+\beta \cdot F_{\text {wave } \text { max }}+\gamma \cdot y_{i, j-1}}}
$$

The results of the fitted Markov models are shown in Figure 6a and 6b respectively, which give the probability of failure as a function of maximum wave force potential, conditioned on whether the seaward structure has survived (green) or failed (red). In both cases, a structure's failure probability is significantly impacted by the performance of its neighboring seaward structure, as illustrated by the large difference between the red and green curves. In particular, for highly exposed structures near the coast with a maximum wave force potential of $300 \mathrm{~m}^{3} / \mathrm{s}^{2}$, the probability of foundation failure given the survival of the seaward structure $(0.83)$ is $13 \%$ lower 
than the failure probability when the seaward structure has failed (0.95). At the same exposure level, the probability of loss failure is $30 \%$ lower. This significant reduction in the vulnerability of inland buildings points to the critical contribution of oceanfront structures on the overall performance of coastal communities. We discuss in greater detail hardening oceanfront structures as a mitigation strategy in the following section.

These results are compared to the vulnerability curve estimated without conditioning on the performance of the seaward structure using logistic regression (Section 2.1). Ignoring the failure of the seaward structure by not conditioning (blue curves) results in an underestimation of a structure's vulnerability (red curves). The magnitude of this underestimation decreases as structures become more exposed. Since the failure of most near-shore structures coincides with the failure of seaward buildings, the estimated failure probability without conditioning (blue curves) is nearly the same as the estimated conditional failure probability (red curves) for structures with high maximum wave force potential. Conversely, ignoring the survival of the seaward structure (blue curves) leads to an overestimation of both physical and loss vulnerability (green curves). This overestimation becomes smaller for less exposed structures further for the coast where less frequent failures tend to coincide with the survival of seaward buildings.

The ordinal Markov model is similarly fit considering four ordinal damage states $(D=4)$ defined according to loss ratio intervals of $0-25 \%, 25-50 \%, 50-75 \%$ and $75-100 \%$. The damage states are coded $0,1,2$ and 3 respectively and related to values of the derived variable $\tilde{\boldsymbol{y}}_{\boldsymbol{i}, \boldsymbol{j}-\mathbf{1}}$ as shown in Table 1 . The model can be summarized as

$$
P\left[Y_{i, j} \leq d \mid Y_{i, j-1}=y_{i, j-1}, F_{\text {wave } \text { max }}\right]=\frac{e^{\alpha_{d}+\beta F_{\text {wave } \max }+\widetilde{y}_{i, j-1}^{\prime} \gamma}}{1+e^{\alpha_{d}+\beta F_{\text {wave } \text { max }}+\tilde{y}_{i, j-1}^{\prime} \gamma}} \quad d=0,1,2
$$

(note that $P\left[Y_{i, j} \leq 3\right]=1$ ). The estimated probability of exceeding a loss ratio level of $25 \%, 50 \%$ and $75 \%$ conditional on $F_{\text {wave,max }}$ and the damage state of the seaward structure is shown in Figure $7 \mathrm{a}, \mathrm{b}$ and $\mathrm{c}$ respectively. (Note that following convention, we show in this plot the probability of exceeding a damage rather than its cumulative probability defined by Eq. 21.) Consistent with the findings from the binary Markov model, the results indicate that the probability of exceeding a damage state has a strong dependence on the damage state of the seaward structure. For an exposed structure with a maximum wave force potential of $300 \mathrm{~m}^{3} / \mathrm{s}^{2}$, for example, the probability of exceeding a loss ratio of $75 \%$ is more than three times greater if the loss ratio of the seaward structures is in the range of $75-100 \%$ compared to $0-25 \%$ (Figure 7c). 
The results of the ordinal Markov model are compared to fragility curves estimated without conditioning on the damage state of the seaward structure using cumulative logit regression (blue curves; Section 2.1). As with the binary case, ignoring the damage state of the building seaward of a structure leads to inaccurate estimation of loss vulnerability.

\subsection{Marginal Model}

We next determine how interdependencies illustrated by the Markov model impact fragility estimation by analyzing foundation and loss failure using the binary marginal model (Section 2.3). This model assumes that only adjacent structures within clusters are correlated and that this correlation is constant. Since there are relatively few clusters in the damage survey, model-based estimates of parameter standard errors are used. The estimated probability of foundation and loss failure and the associated statistical uncertainty (i.e. 95\% confidence interval) are shown in green in Figure $8 \mathrm{a}$ and $8 \mathrm{~b}$ respectively. In each subfigure the estimated correlation of structures in adjacent rows $\hat{\rho}$ is also shown. We compare these results to the estimated failure probability and uncertainty (shown in red) when assuming the independence of damage observations using standard logistic regression (Section 2.1).

The estimated failure probability as function of maximum wave force potential when assuming independence (solid red curve) and considering correlation (solid green curve) is essentially the same for both foundation and loss failure. Recalling that for a Bernoulli variable the probability of observing failure $\left(P\left[Y_{i, j}=1 \mid F_{\text {wave,max }}\right]\right)$ is equivalent to the expected value of the response $\left(E\left[Y_{i, j} \mid F_{\text {wave,max }}\right]\right)$, the similarity between the two curves indicates that in this case performance interdependencies have little effect on statistical estimation of the mean (i.e. average) failure response as a function of the hazard covariate. Comparing the shaded confidence bands, however, shows that the statistical uncertainty in this estimate increases when correlation is accounted for. For an exposed near-shore structure with a maximum wave force potential of 300 $\mathrm{m}^{3} / \mathrm{s}^{2}$, for example, the confidence interval for the probability of foundation failure when considering correlation ([.85, .99]) is $40 \%$ larger relative to when correlation is neglected ([.89, .99]). This increased uncertainty implies that the data yields less information about how wave force potential relates to performance than one is initially led to believe when neglecting correlation. Vulnerability estimation when assuming independence wrongly attributes all of the variation in the damage response to changes in the hazard covariate. In reality, however, a portion of this variability is a consequence of performance interdependencies, which when accounted for, increases the uncertainty of how the hazard covariate in fact relates to the 
response. This increased uncertainty is a typical finding in previous studies using marginal models to consider correlation in longitudinal data [16].

\section{Implications}

As a result of physical interaction between buildings during storm surge, a structure's performance is not only a function of its own ability to resist damage but also the ability of surrounding buildings to do likewise. In this section we discuss the implications of interdependencies on various aspects of storm surge risk mitigation. Although we emphasize storm surge, this discussion applies more generally to any hazard where interdependencies between residential structures may arise.

\subsection{Insurance Pricing}

Performance interdependencies are particularly relevant for insurance pricing by FEMA's National Flood Insurance Program (NFIP), which is the primary mechanism for quantifying and managing coastal risk. Although homeowners may pursue costly retrofits to mitigate their vulnerability and reduce their premiums (e.g. by elevating their structure on piles), homeowners and FEMA may over or underestimate the actual risk reduction due to these actions if the actions of surrounding homeowners are not considered. How to incorporate these interdependencies into sensible insurance pricing strategies is further complicated by the fact that decision making amongst homeowners is itself oftentimes interdependent, with homeowners hesitant to pursue mitigation unless they see others in the community doing the same [42].

The system-like behavior of structures within coastal communities means that returns on mitigation measures may be greatest when coordinated rather than taken wholly independently by homeowners. Recognizing the importance of a more comprehensive approach to coastal management, FEMA has promoted community-wide mitigation measures through its Community Rating System (CRS) program, offering premium discounts for communities exceeding minimum design requirements [43]. CRS policies incentivizing higher regulatory standards, such as freeboard requirements and open space protection, have helped reduce flood damage since the program's inception in 1990 [44]. Building on this holistic approach, the Markov-based vulnerability modeling presented in this study allows stakeholders to weigh potential scenarios a structure may encounter and make decisions accordingly.

\subsection{Structural Design}


An active research need is incorporating and standardizing the treatment of performance interdependencies in codified structural design [45]. One approach to implicitly handle storm surge interdependencies is by incorporating interaction effects into minimum design loads using ASCE 7-10 [46]. As is the case with design wind loads which are modified to account for the built environment, structural shielding due to permanent obstructions and/or the proximity of structures can be considered using load reduction factors. Furthermore, a greater emphasis should be placed on debris impact loads and their contribution to flood load combinations. Incorporating shielding and debris effects in structural design would of course be contingent on an improved physical understanding of surge-structure interaction, which fortunately has seen promising progress in recent years.

\subsection{Mitigation Strategies}

A critical aspect of community vulnerability is robustness, or the inherent ability of a community as a system to resist external demands [47]. Although we have emphasized the detrimental effects of interdependencies, the integrated performance of structures also provides a powerful means of improving community robustness since mitigation actions taken by individual homeowners have residual benefits to the community as a whole. In particular, the significant impact of oceanfront structures on the vulnerability of inland buildings points to hardening these structures (by elevating them off the ground or by increasing their resistance) as an attractive approach to improving community performance. Although this would not prevent large losses due to flood inundation, it would help reduce costly structural damage to inland buildings caused by wave and debris impact.

Indeed, the benefits of hardening oceanfront structures can be seen in Ortley Beach's performance during Hurricane Sandy. Figure 9 shows the loss ratios of damaged structures in the community separated into two regions. The region labeled as ' $\mathrm{A}$ ' contains the surveyed structures used in this study. The neighboring region labeled ' $\mathrm{B}$ ' contains additional structures assessed from the damage survey. With the age and type of structures uniformly distributed across both regions, the notable difference in their relative performance points to built environment features influencing the overall vulnerability of the regions. A closer inspection reveals characteristic differences in the oceanfront structures in the two regions, also shown in Figure 9. While those in region ' $A$ ' were typical wood frame residential structures, those in region ' $\mathrm{B}$ ' were large multi-family and commercial structures elevated on piles. Despite these latter structures sustaining significant damage to their building envelopes, their overall survival 
compared to the complete destruction of those in region ' $A$ ' helps explain the notable difference in structural performance between the two regions.

\subsection{Community risk and resilience}

Interdependencies broaden the scope of coastal risk assessment from interest solely in the performance of individual structures to the performance of communities as a whole. This transition in turn emphasizes the need for improved community resilience, or "the ability to prepare and plan for, absorb, recover from, and more successfully adapt to adverse events" [48]. The statistical approaches presented in this study offer practical means of bridging the gap between risk and resilience at the individual building and community levels. Specifically, conditional vulnerability and fragility curves from the Markov models distinguish the different contributions of hazard exposure and community interdependencies on structural performance. Furthermore, traditional unconditional vulnerability curves from the marginal model relate performance to hazard exposure while also implicitly incorporating the effects of interdependencies (through estimation of the intra-cluster correlation $\widehat{\boldsymbol{R}}_{i}$ ).

One application of particular interest for this community-level approach is assessing the performance of coastal asset portfolios, which involves estimating the expected value and variance of total portfolio losses [49]. Since the variance of the sum of a set of positively correlated random variables is greater than the sum of the variances (when assuming independence), neglecting correlation between assets may underestimate the true variance of portfolio losses. A similar underestimation has been shown to be the case when neglecting the correlated performance of structures during earthquakes due to the spatial correlation of seismic loads [50]. Vulnerability curves and associated correlation estimates from the marginal model offer an attractive means of incorporating these interdependencies into coastal portfolio loss estimation.

\section{Conclusion}

In this study, two statistical approaches for estimating vulnerability and inferring interdependencies from observed damage are proposed. To determine how interdependencies contribute to vulnerability, we present a Markov model which treats interaction as an additional demand parameter. This analysis yields vulnerability and fragility curves relating a structure's performance to hazard intensity conditional on the performance of surrounding buildings. To determine how interdependencies impact the statistical relationship between performance and hazard exposure, we present a marginal model which incorporates correlation between damage 
observations into the estimation of regression parameters. This analysis yields vulnerability and fragility curves which implicitly include the effects of interdependencies. A case study of a coastal community impacted by Hurricane Sandy indicates that the performance of a structure is strongly dependent on the performance of the structure immediately seaward. Furthermore, accounting for this dependence increases the uncertainty when estimating the relationships between performance and hazard parameters. These findings demonstrate that coastal residential communities comprise systems whose overall reliability reflects the integrated performance of individual structures. Engineering and policy actions which ignore this system-like behavior potentially neglect a critical aspect of a structure's (and a community's) vulnerability.

Important future work concerning performance interdependencies remains to be explored. In addition to using larger surveys of surge damage from different regions and events, future studies can investigate how built environment features such as the proximity of structures within a community influence interdependencies. Furthermore, the modeling approaches presented here can be generalized to other natural hazards exhibiting interaction mechanisms in multiple directions. Reviews of spatial extensions for both models can be found in Besag [51] and Albert \& McShane [52].

\section{Acknowledgements}

This research was supported by National Science Foundation Grant 1520683. The authors would like to thank Dr. Andrew Kennedy for providing data from the hydrodynamic simulation of storm surge inundation in Ortley Beach.

\section{References}

[1] Rinaldi SM, Peerenboom JP, Kelly TK. Identifying, understanding, and analyzing critical infrastructure interdependencies. Cont Syst, IEEE. 2001;21:11-25.

[2] Dobson I, Carreras BA, Lynch VE, Newman DE. Complex systems analysis of series of blackouts: cascading failure, critical points, and self-organization. Chaos. 2007;17:026103. [3] Ouyang M. Review on modeling and simulation of interdependent critical infrastructure systems. Reliab Eng Syst Safety. 2014;121:43-60.

[4] Dueñas-Osorio L, Craig JI, Goodno BJ. Seismic response of critical interdependent networks. Earthquake Engng Struct Dyn. 2007;36:285-306.

[5] Anagnostopoulos SA. Pounding of buildings in series during earthquakes. Earthquake Engng Struct Dyn. 1988;16:443-56.

[6] Minor JE. Windborne debris and the building envelop. J Wind Eng Ind Aerodyn. 1994;53:207-27.

[7] Mousavi S, Bagchi A, Kodur VK. Review of post-earthquake fire hazard to building structures. Can J Civ Eng. 2008;35:689-98. 
[8] Jakob M, Hungr O. Debris-flow hazards and related phenomena. Germany: Springer Berlin Heidelberg; 2005.

[9] Charvet I, Suppasri A, Imamura F. Empirical fragility analysis of building damage caused by the 2011 Great East Japan Tsunami in Ishinomaki City using ordinal regression, and influence of key geographical features. Stoch Env Res Risk A. 2014;28:1853-67.

[10] Hatzikyriakou A, Lin N, Gong J, Xian S, Hu X, Kennedy A. Component-based vulnerability analysis of storm surge damage to residenetial structures from Hurricane Sandy using manual and LiDAR surveys. Nat Hazards Rev. 2015.

[11] Federal Emergency Management Agency. Hurricane Sandy in New Jersey and New York (FEMA P-942). Mitigation Assessment Team Report 2013.

[12] Tomiczek T, Kennedy A, Zhang Y, Owensby M, Hope M, Lin N, et al. Hurricane damage classification methodology and fragility functions derived from Hurricane Sandy's effects in coastal New Jersey. J Waterw Port Coast Ocean Eng. 2016. (in review)

[13] Lin N, Vanmarcke E, Yau S-C. Windborne debris risk analysis - Part II. Application to structural vulnerability modeling. Wind Struct. 2010;13:207-20.

[14] Vickery P, Lin J, Skerlj P, Twisdale L, Huang K. HAZUS-MH Hurricane Model

Methodology. I: Hurricane Hazard, Terrain, and Wind Load Modeling. Nat Hazards Rev. 2006;7:82-93.

[15] Reese S, Bradley BA, Bind J, Smart G, Power W, Sturman J. Empirical building fragilities from observed damage in the 2009 South Pacific tsunami. Earth-Sci Rev. 2011;107:156-73.

[16] Le Cessie S, Van Houwelingen J. Logistic regression for correlated binary data. J Roy Stat Soc C-App. 1994;43:95-108.

[17] Agresti A. Analysis of ordinal categorical data. 2 ed. Hoboken, New Jersey: John Wiley \& Sons; 2010.

[18] Zeger SL, Liang KY. An overview of methods for the analysis of longitudinal data. Stat Med. 1992;11:1825-39.

[19] Diggle P, Heagerty P, Liang K-Y, Zeger S. Analysis of longitudinal data. 2 ed. Great Britian: Oxford University Press; 2002.

[20] Chadoeuf J, Pierrat J, Nandris D, Geiger J-P, Nicole M. Modeling rubber tree root disease epidemics with a Markov spatial process. Forest Sci. 1993;39:41-54.

[21] Hubbell SP, Ahumada JA, Condit R, Foster RB. Local neighborhood effects on long-term survival of individual trees in a Neotropical forest. Ecol Res. 2001;16:859-75.

[22] Rahman S, Grigoriu M. Markov model for seismic reliability analysis of degrading structures. J Struct Eng. 1993;119:1844-65.

[23] Baik H-S, Jeong HS, Abraham DM. Estimating transition probabilities in Markov chainbased deterioration models for management of wastewater systems. J Water Res Pl Ma. 2006;132:15-24.

[24] Hardin JW, Hilbe JM. Generalized Estimating Equations. 2nd ed. Boca Raton: CRC Press; 2013.

[25] Gregersen IB, Madsen H, Rosbjerg D, Arnbjerg-Nielsen K. A spatial and nonstationary model for the frequency of extreme rainfall events. Water Resour Res. 2013;49:127-36.

[26] Zeger SL, Liang K-Y, Albert PS. Models for longitudinal data: a generalized estimating equation approach. Biometrics. 1988:1049-60.

[27] R Development Core Team. R: A language and environment for statistical computing.

Vienna, Austria: R Foundation for Statistical Computing; 2015.

[28] Agresti A. Categorical data analysis. 3 ed. Hoboken, New Jersey: John Wiley \& Sons; 2013. 
[29] Lallemant D, Kiremidjian A, Burton H. Statistical procedures for developing earthquake damage fragility curves. Earthquake Engng Struct Dyn. 2015;44:1373-89.

[30] Bartimote-Aufflick K, Thomson PC. The analysis of ordinal time-series data via a transition (Markov) model. J Appl Stat. 2011;38:1883-97.

[31] Hu FB, Goldberg J, Hedeker D, Flay BR, Pentz MA. Comparison of population-averaged and subject-specific approaches for analyzing repeated binary outcomes. Am J Epidemiol. 1998;147:694-703.

[32] Wang M. Generalized Estimating Equations in Longitudinal Data Analysis: A Review and Recent Developments. Adv Stat. 2014;2014.

[33] Zeger SL, Liang K-Y. Longitudinal data analysis for discrete and continuous outcomes. Biometrics. 1986:121-30.

[34] Myers RH, Montgomery DC, Vining GG, Robinson TJ. Generalized linear models: with applications in engineering and the sciences. Hoboken, New Jersey: John Wiley \& Sons; 2012. [35] Horton NJ, Lipsitz SR. Review of software to fit generalized estimating equation regression models. Am Stat. 1999;53:160-9.

[36] Blake ES, Kimberlain TB, Berg RJ, Cangialosi J, Beven II JL. Tropical Cyclone Report: Hurricane Sandy (AL182012). National Hurricane Center; 2013.

[37] O'Dea C. Interactive map: Assessing damage from Superstorm Sandy. njspotlight.com; 2013.

[38] Xian S, Lin N, Hatzikyriakou A. Storm surge damage to residential areas: a quantitative analysis for Hurricane Sandy in comparison with FEMA flood map. Nat Hazards. 2015:1-22. [39] Dall'Osso F, Maramai A, Graziani L, Brizuela B, Cavalletti A, Gonella M, et al. Applying and validating the PTVA-3 Model at the Aeolian Islands, Italy: assessment of the vulnerability of buildings to tsunamis. Nat Hazard Earth Sys. 2010;10:1547-62.

[40] Shim JS, Kim J, Kim DC, Heo K, Do K, Park SJ. Storm surge inundation simulations comparing three-dimensional with two-dimensional models based on Typhoon Maemi over Masan Bay of South Korea. J Coastal Res. 2013.

[41] Federal Emergency Management Agency. Substantial Improvement/ Substantial Damage Desk Reference (FEMA P-758). 2010.

[42] Kunreuther H. Disaster mitigation and insurance: Learning from Katrina. Ann Am Acad Polit Ss. 2006;604:208-27.

[43] Federal Emergency Management Agency. Community Rating System: A Local Official's Guide to Saving Lives, Preventing Property Damage and Reducing the Cost of Flood Insurance (FEMA 573). 2006.

[44] Highfield W, Brody S. Evaluating the Effectiveness of Local Mitigation Activities in Reducing Flood Losses. Nat Hazards Rev. 2013;14:229-36.

[45] McAllister T. Research Needs for Developing a Risk-Informed Methodology for Community Resilience. J Struct Eng. 2015.

[46] American Society of Civil Engineers. Minimum Design Loads for Buildings and Other Structures 7-10. Reston, VA: American Society of Civil Engineers; 2013.

[47] Bruneau M, Chang SE, Eguchi RT, Lee GC, O'Rourke TD, Reinhorn AM, et al. A framework to quantitatively assess and enhance the seismic resilience of communities. Earthq Spectra. 2003;19:733-52.

[48] The National Academies. Disaster resilience: a national imperative. Washington, D.C.:

National Academy of Sciences; 2012. 
[49] Schubert M, Faber MH. Common cause effects in portfolio loss estimation. Struct Infrastruct Eng. 2012;8:497-506.

[50] Park J, Bazzurro P, Baker J. Modeling spatial correlation of ground motion intensity measures for regional seismic hazard and portfolio loss estimation. Applications of statistics and probability in civil engineering. London: CRC Press; 2007.

[51] Besag J. Spatial interaction and the statistical analysis of lattice systems. J Roy Stat Soc B Met. 1974;36:192-236.

[52] Albert PS, McShane LM. A generalized estimating equations approach for spatially correlated binary data: applications to the analysis of neuroimaging data. Biometrics.

1995;51:627-38.

\section{Tables and Figures}

\begin{tabular}{cc}
$y_{i, j-1}$ & $\widetilde{\boldsymbol{y}}_{\boldsymbol{i} \boldsymbol{j} \mathbf{j - 1}}$ \\
\hline 0 & $(1,1,1)$ \\
\hline 1 & $(0,1,1)$ \\
\hline 2 & $(0,0,1)$ \\
\hline 3 & $(0,0,0)$
\end{tabular}

Table 1 - Relation between damage state $y_{i, j-1}$ and the derived variable $\widetilde{\boldsymbol{y}}_{i, j-\mathbf{1}}$ for four ordinal damage states labeled $0,1,2$ and 3 .

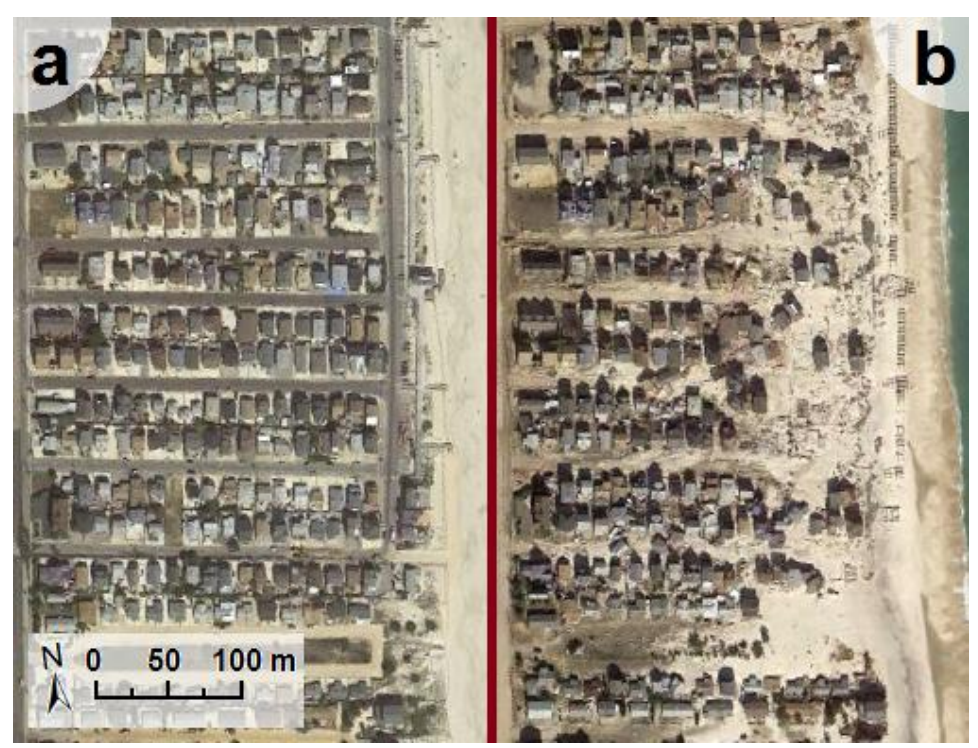

Figure 1 - Plan view of surveyed structures in Ortley Beach, New Jersey. Satellite images (a) before and (b) after Hurricane Sandy were taken on September 20, 2010 and November 3, 2012, respectively. (Map Data: (C) 2010, 2012 Google) 


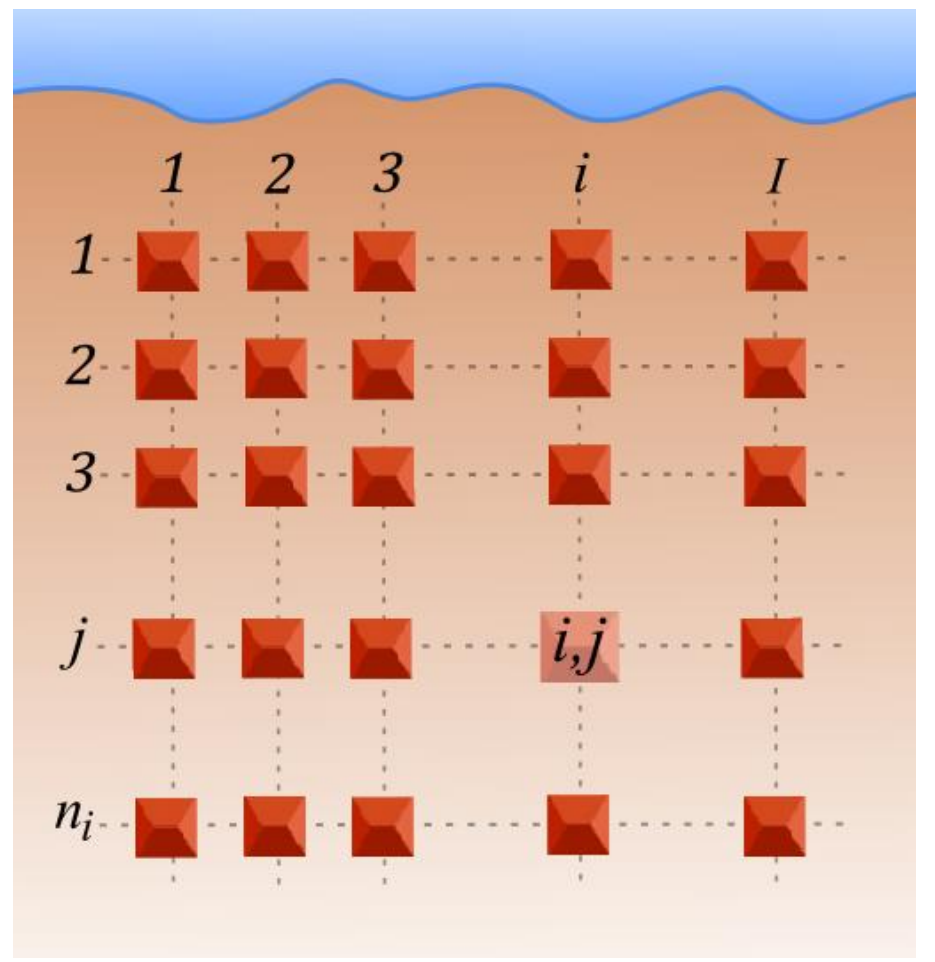

Figure 2 - Indexing of structures in a hypothetical coastal community according to their cluster $i$ and row from the coast $j$. The community's coastline is represented by the blue curved line.

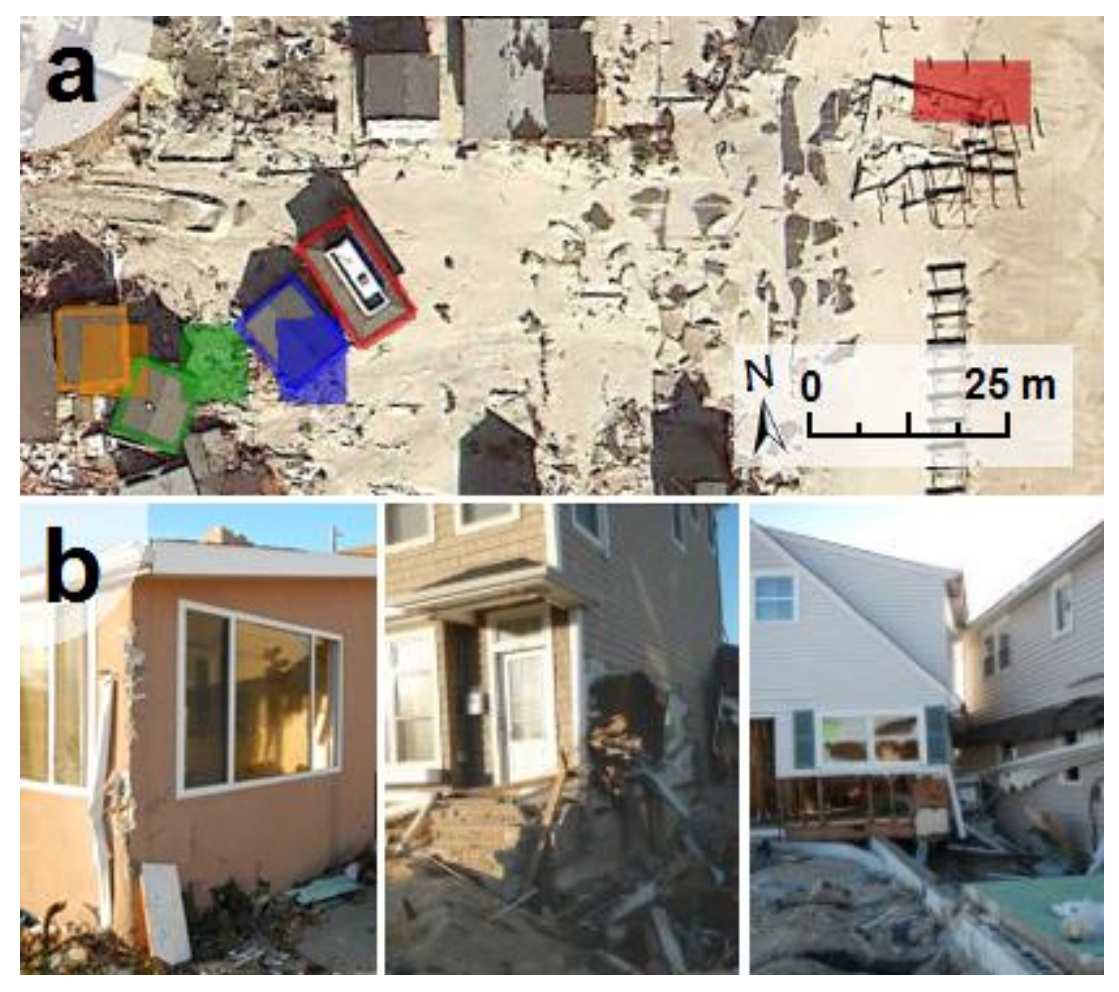

Figure 3 - Structural damage due to waterborne debris. (a) Chain reaction of foundation failures due to structures being carried inland by storm surge. Colored polygons represent the original footprints of the outlined structures. (Map Data: C 2010 Google) (b) Structures with light, 
moderate and severe damage due to waterborne debris impact. Both subfigures reproduced from Hatzikyriakou et al. [10].
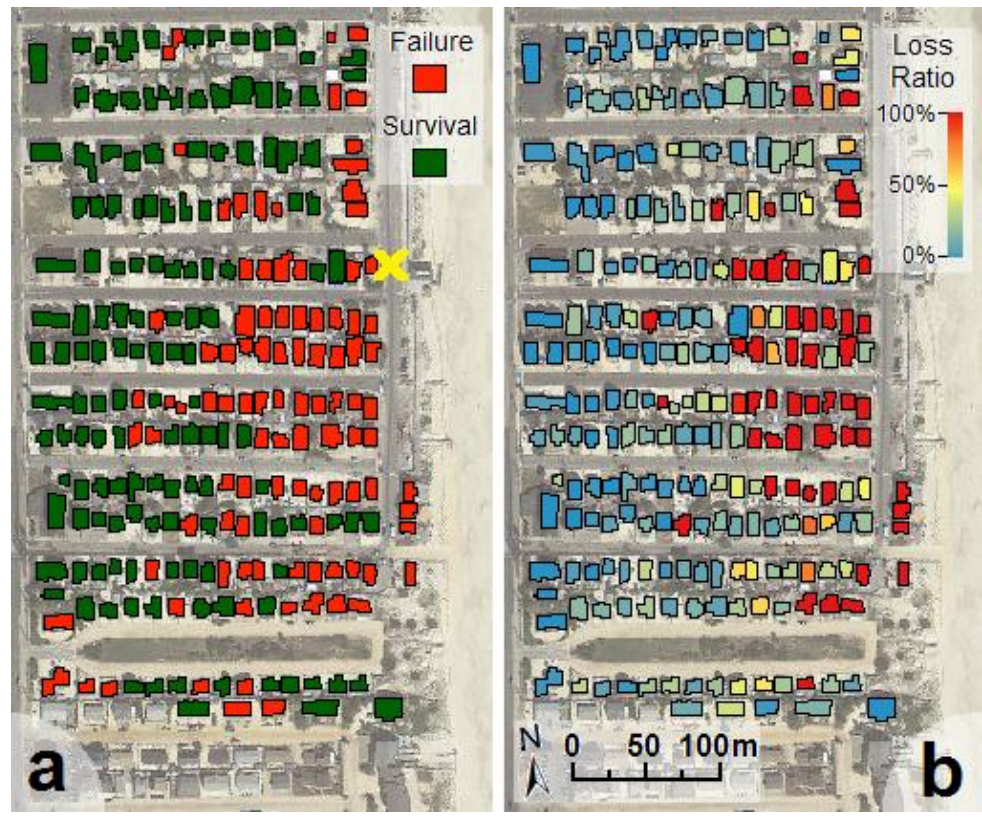

Figure 4 - Assessed performance of surveyed structures in Ortley Beach, New Jersey. (a) Foundation performance with red and green polygons representing structures with foundation failure and survival respectively. (Map Data: (C) 2010 Google; reproduced using data from Hatzikyriakou et al. [10]) (b) Economic performance with structures shaded according to assessed loss ratio. (Map Data: (C) 2010 Google; reproduced using data from Xian et al. [38]) 

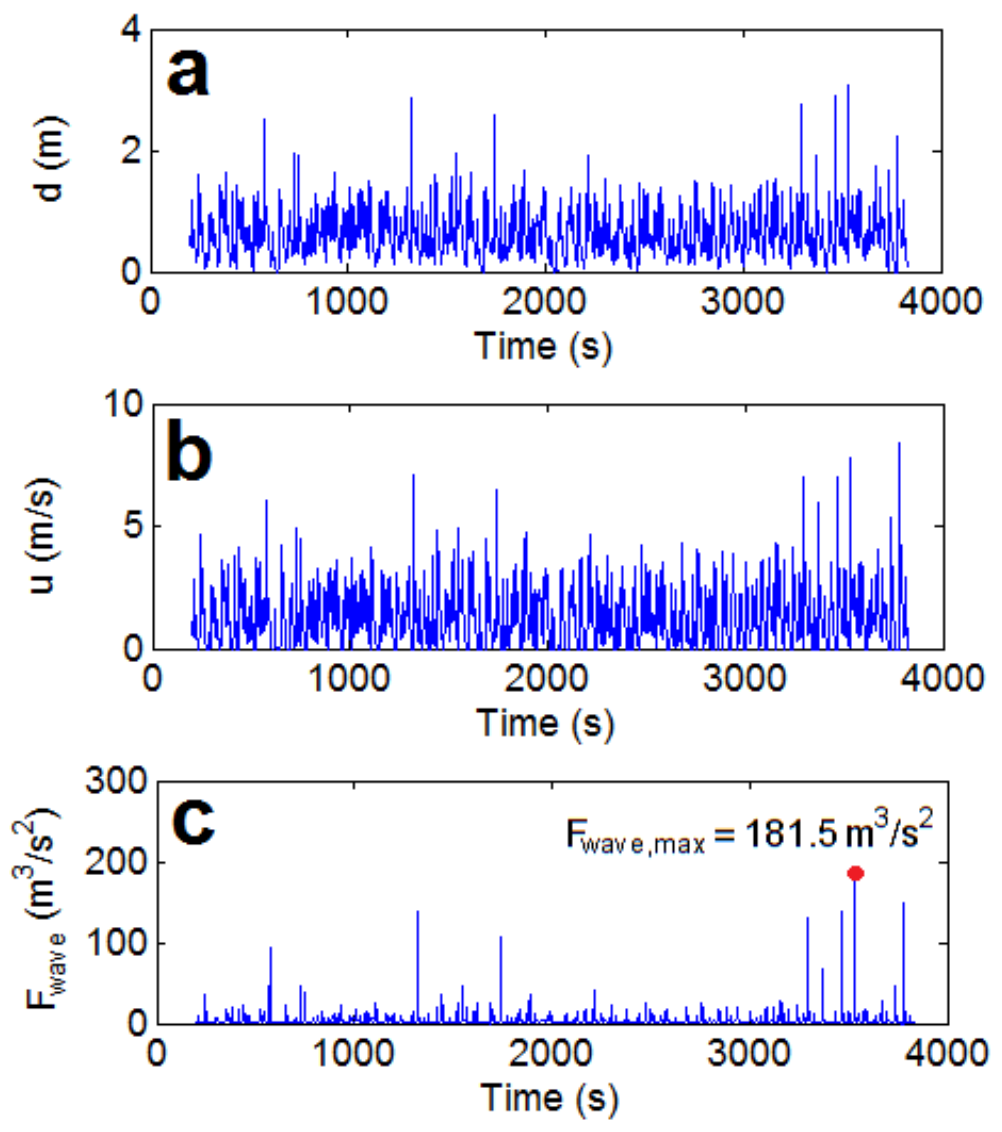

Figure 5 - Hydrodynamic simulation of storm surge inundation in Ortley Beach. Time histories of (a) inundation depth, (b) on-shore surge velocity and (c) wave force potential are shown for the structure marked with a yellow ' $x$ ' in Figure 4a. The maximum wave force potential for the structure is highlighted in (c). (Simulation data from Tomiczek et al. [12])
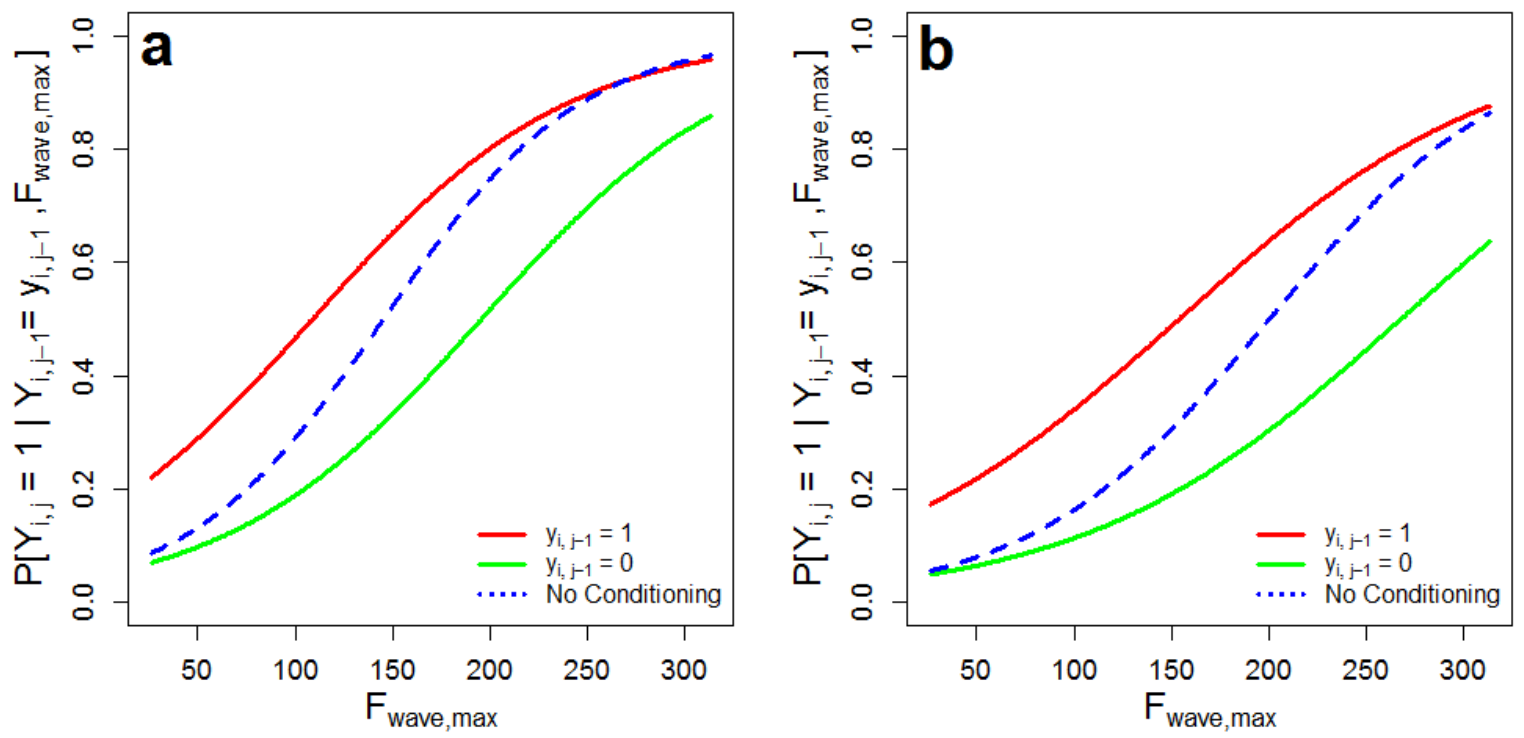
Figure 6 - Conditional vulnerability curves using binary Markov model. Probability of (a) foundation and (b) loss failure as a function of maximum wave force potential, conditional on the failure (red curves) and survival (green curves) of the structure immediately seaward. These results are compared to the probability of failure estimated without conditioning (dashed blue curves) using logistic regression.
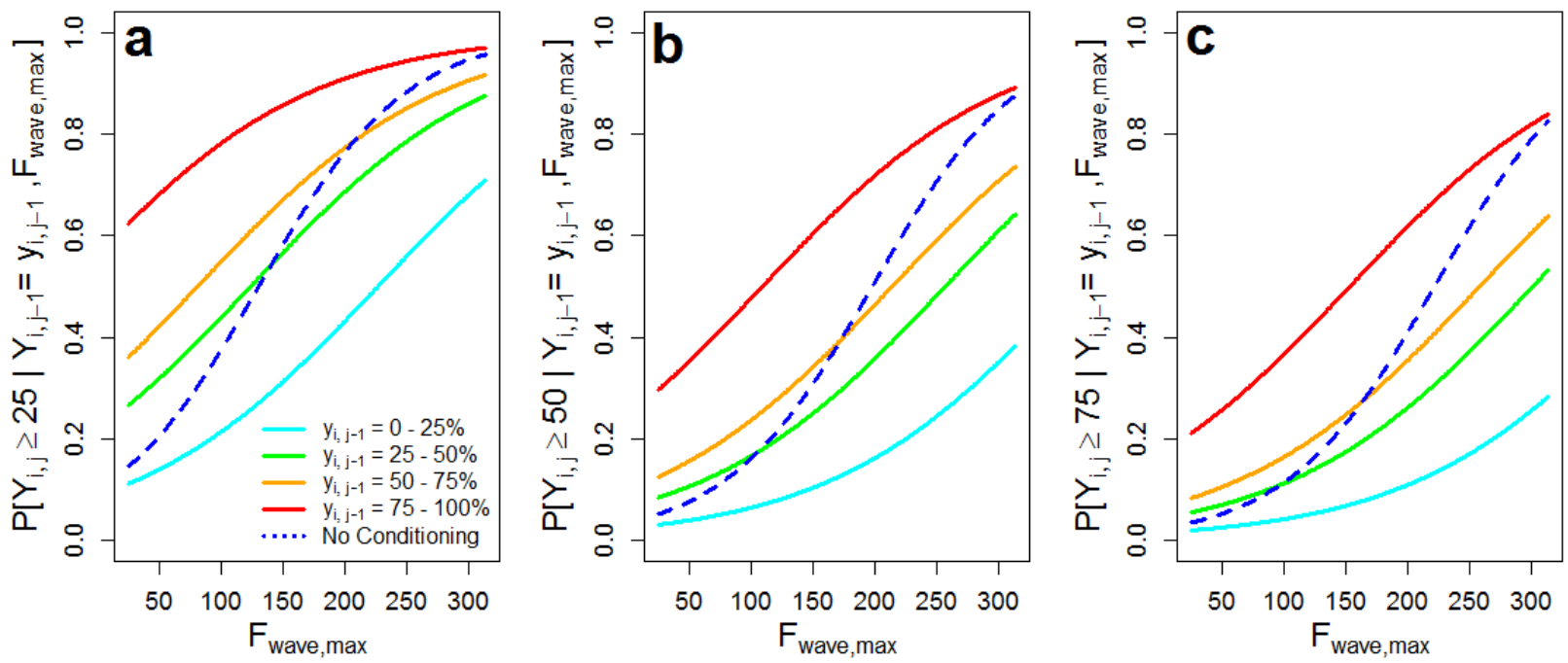

Figure 7 - Conditional fragility curves using ordinal Markov model. Probability of equaling or exceeding a loss ratio of (a) $25 \%$, (b) $50 \%$ and (c) $75 \%$ as a function of maximum wave force potential, conditional on the loss ratio damage state of the structure immediately seaward. These results are compared to the exceedance probability estimated without conditioning (dashed blue curves) using cumulative logit regression.
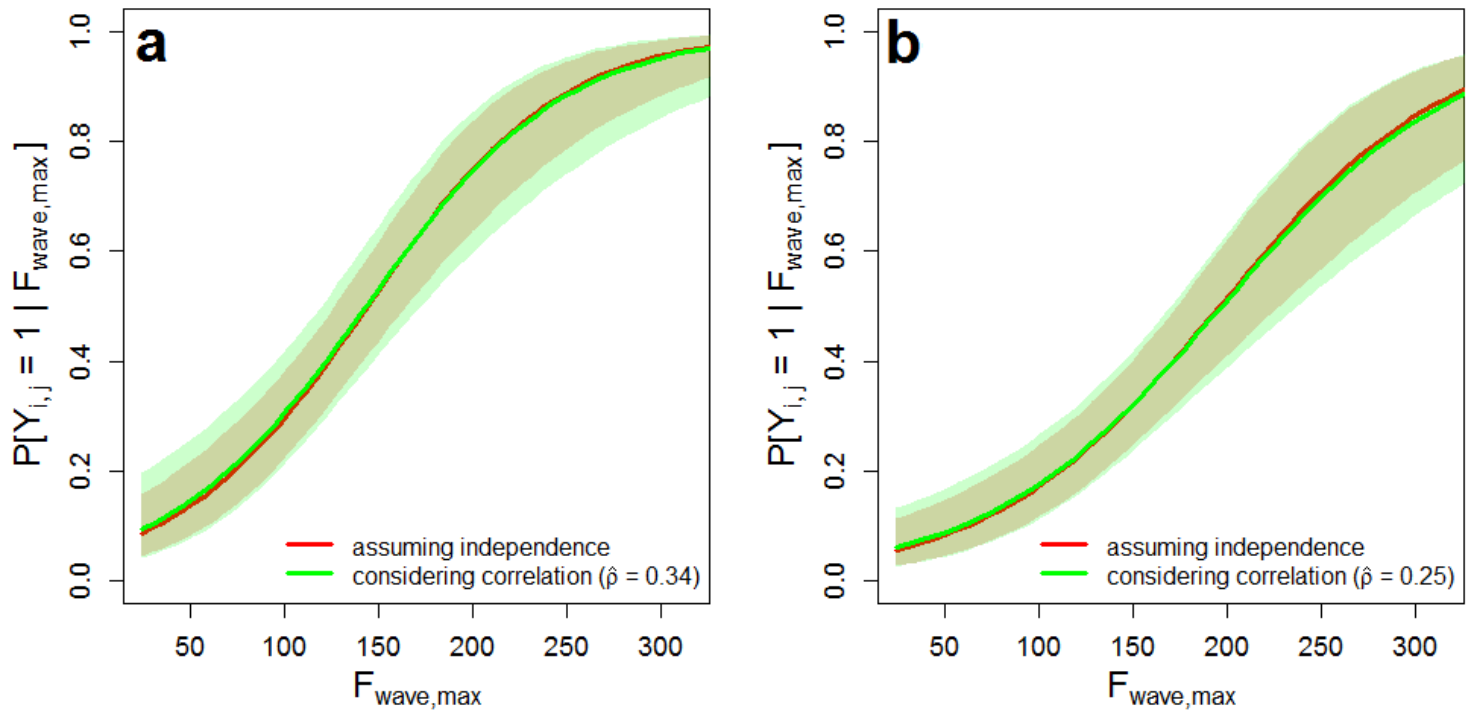

Figure 8 - Vulnerability curves incorporating correlation between damage observations using marginal model. The probability of (a) foundation and (b) loss failure and the associated $95 \%$ confidence intervals when considering correlation with marginal model are shown in green. These results are compared to the failure probabilities and $95 \%$ confidence intervals when 
assuming independence using logistic regression, shown in red. (Note that the shaded green confidence interval is semi-transparent.)

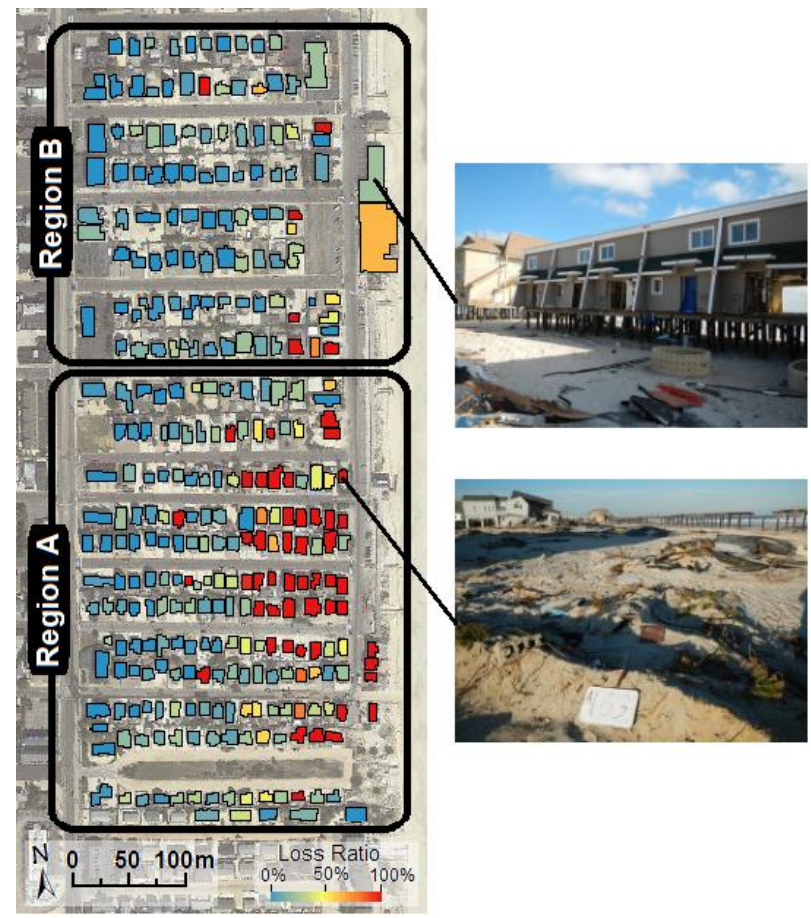

Figure 9 - Impact of oceanfront structures on overall community performance during storm surge. Structures in Ortley Beach shaded according to their assessed loss ratio are divided into two regions. Region 'A' contains structures used as part of this study and Region 'B' contains additional surveyed buildings in the community. Photographs of oceanfront structures in both regions are likewise shown. (Figure reproduced from Hatzikyriakou et al. [10]) 